\title{
TRAP-seq identifies cystine/glutamate antiporter as a driver of recovery from liver injury
}

\author{
Amber W. Wang, ${ }^{1}$ Kirk J. Wangensteen, ${ }^{1,2}$ Yue J. Wang, ${ }^{1}$ Adam M. Zahm, ${ }^{1}$ Nicholas G. Moss, ${ }^{1}$ Noam Erez, ${ }^{1,2}$ and Klaus H. Kaestner \\ 'Department of Genetics and ²Department of Medicine, University of Pennsylvania, Philadelphia, Pennsylvania, USA.
}

\begin{abstract}
Understanding the molecular basis of the regenerative response following hepatic injury holds promise for improved treatment of liver diseases. Here, we report an innovative method to profile gene expression specifically in the hepatocytes that regenerate the liver following toxic injury. We used the $\mathrm{Fah}^{-/-}$mouse, a model of hereditary tyrosinemia, which conditionally undergoes severe liver injury unless fumarylacetoacetate hydrolase (FAH) expression is reconstituted ectopically. We used translating ribosome affinity purification followed by high-throughput RNA sequencing (TRAPseq) to isolate mRNAs specific to repopulating hepatocytes. We uncovered upstream regulators and important signaling pathways that are highly enriched in genes changed in regenerating hepatocytes. Specifically, we found that glutathione metabolism, particularly the gene SIc7a11 encoding the cystine/glutamate antiporter (xCT), is massively upregulated during liver regeneration. Furthermore, we show that SIc7a11 overexpression in hepatocytes enhances, and its suppression inhibits, repopulation following toxic injury. TRAP-seq allows cell type-specific expression profiling in repopulating hepatocytes and identified xCT, a factor that supports antioxidant responses during liver regeneration. xCT has potential as a therapeutic target for enhancing liver regeneration in response to liver injury.
\end{abstract}

\section{Introduction}

The liver is the main metabolic organ in the body; it is the nexus for homeostasis of carbohydrates, proteins, and lipids, and it eliminates waste products by oxidation and reduction, conjugation, and excretion into the bile. As such, the liver is exposed to environmental toxins that can severely damage hepatocytes and cause acute liver failure (1). Animals have conserved the ability to regenerate the liver parenchyma upon damage (2) and to restore full mass and function even with the loss of up to $75 \%$ of hepatocytes (3).

Liver cells in adult animals are normally quiescent and divide infrequently. With acute tissue damage, however, mature hepatocytes and cholangiocytes enter the cell cycle and divide (4). In addition, hepatocyte proliferation occurs after partial hepatectomy ( $\mathrm{PHx})$, a noninflammatory liver regeneration model in which up to two-thirds of the liver is removed (5). In rodents, this leads to cell division in most hepatocytes within hours and expansion of the remnant organ over the course of 1 to 2 weeks, until the entire mass of the liver is restored. Because PHx is relatively easily carried out in rodents, it has been used to study liver regeneration in mice for decades $(5,6)$. In fact, many studies have profiled changes in gene expression during regeneration, and a number of important genes and pathways have been identified (7-9). The common theme from these studies is that cell-cycle genes are upregulated and metabolic genes are downregulated as hepatocytes divide to recover from $\mathrm{PHx}$.

Related Commentary: p. 2192

Authorship note: AWW and KJW contributed equally to this work.

Conflict of interest: The authors have declared that no conflict of interest exists.

Submitted: May 12, 2017; Accepted: March 6, 2018.

Reference information: / Clin Invest. 2018;128(6):2297-2309.

https://doi.org/10.1172/JCI95120.
Other paradigms to study liver regeneration utilize injury models involving treatment of animals with hepatotoxins to examine the expression changes of injured liver tissue taken en bloc (10-12). However, until now there has been no methodology to distinguish the responses of the healthy, repopulating liver cells from those of damaged hepatocytes and inflammatory cells. In clinically relevant hepatic injury, a minority of cells may be protected from the initial insult and thus poised to drive repopulation $(13,14)$. It is therefore important to establish which genes in the repopulating hepatocyte drive regeneration in the setting of widespread injury.

The mouse model of hereditary tyrosinemia, an inborn error of tyrosine metabolism caused by a deficiency of fumarylacetoacetate hydrolase (FAH) enzyme (14), is useful for studying the mechanisms of liver regeneration, since repopulating hepatocytes can be labeled as they divide to restore liver function after injury. Homozygously null $\left(\mathrm{Fah}^{-/}\right)$mice die at birth with hepatic dysfunction from toxic metabolites but can be maintained in a healthy state by the drug 2-(2-nitro-4-trifluoromethylbenzoyl)-1,3-cyclohexanedione (NTBC) (14). Alternatively, gene therapy that restores FAH expression can normalize tyrosine catabolism within hepatocytes and allow liver repopulation by the corrected cells upon NTBC removal (15). Our previous work also demonstrated that transgenes can be coexpressed with FAH and can be used to genetically trace repopulating hepatocytes over time $(15,16)$.

Here, we use translating ribosome affinity purification (TRAP) (17) followed by high-throughput RNA sequencing (TRAP-seq) to profile the gene expression pattern specific to repopulating hepatocytes. Slc7a11, encoding the cystine/glutamate antiporter $(\mathrm{xCT})$, was massively activated in regenerating hepatocytes. $\mathrm{xCT}$ imports cystine as a precursor for glutathione (GSH) synthesis $(18,19)$. We show that ectopic expression of $\mathrm{xCT}$ promotes liver repopulation, whereas CRISPR/Cas9-mediated 
A
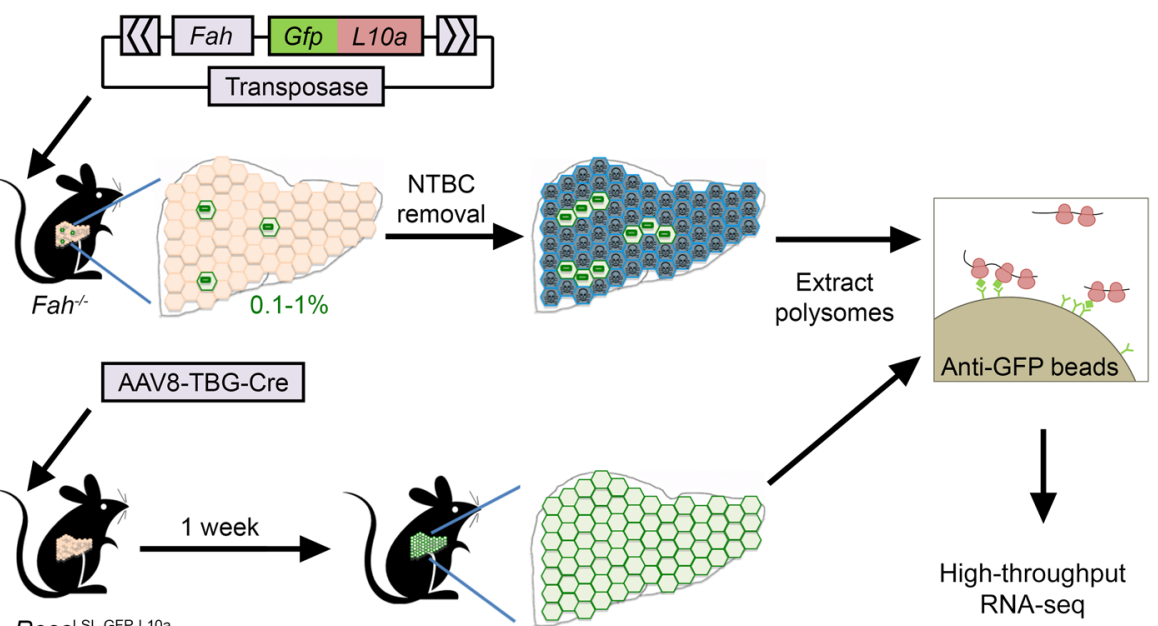

Rosa ${ }^{\text {LSL-GFP-L10a }}$ polysomes

High-throughput RNA-seq

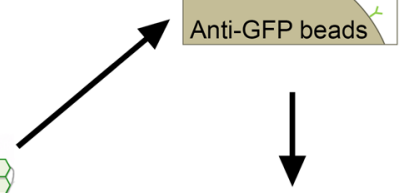

B

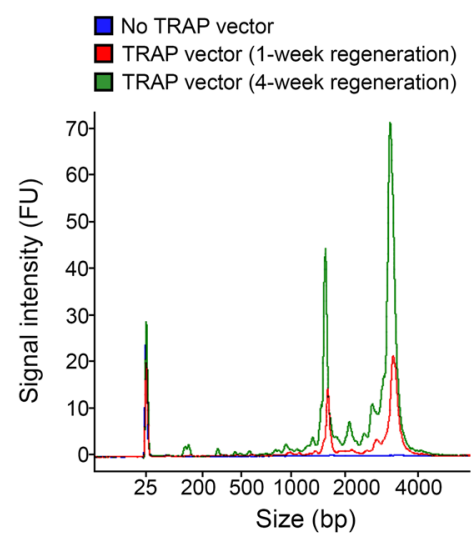

C
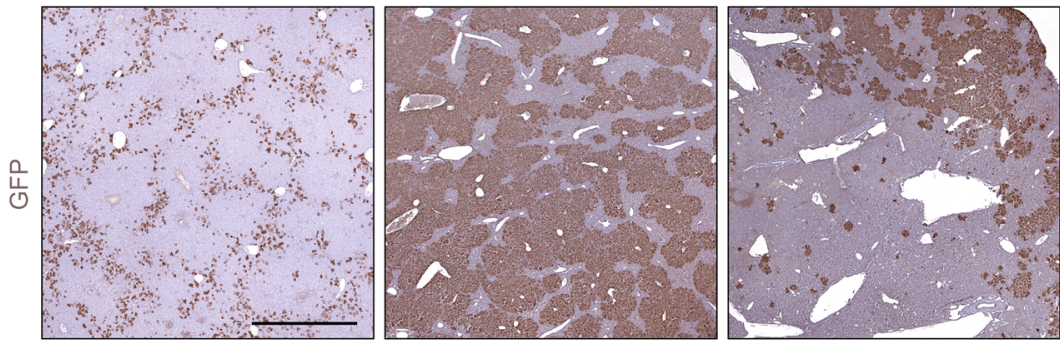

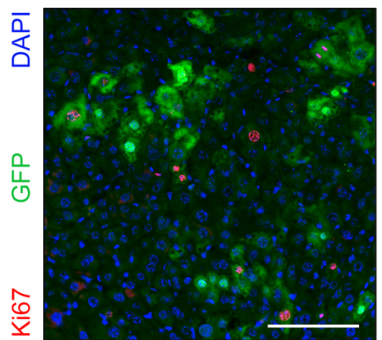

1-week regeneration

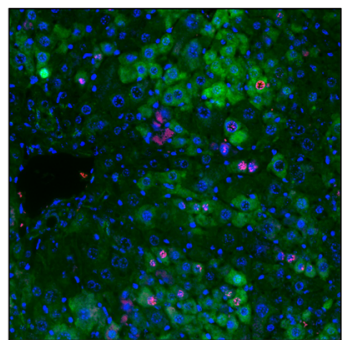

4-week regeneration

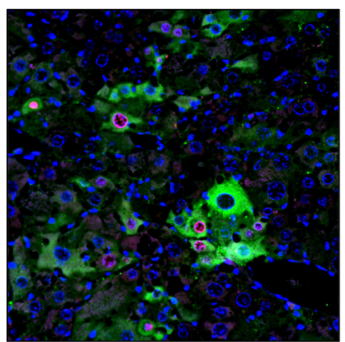

4-week regeneration after severe injury
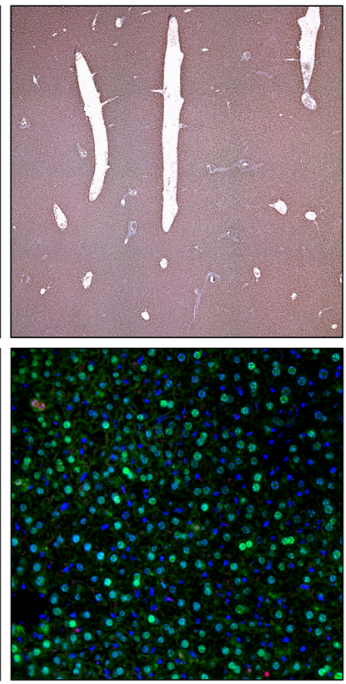

+ AAV8-TBG-Cre
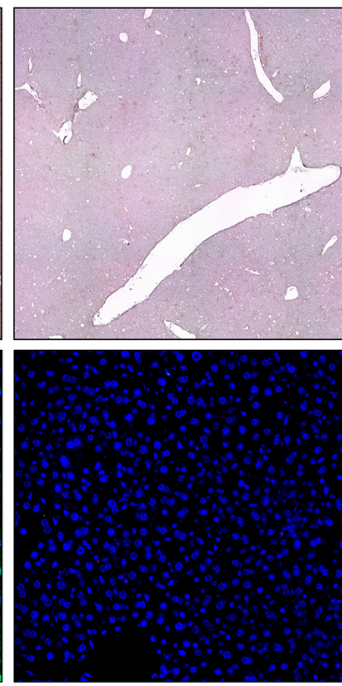

-AAV8-TBG-Cre

Figure 1. TRAP enables cell type-specific isolation of RNA from quiescent and repopulating hepatocytes. (A) The approach for isolating repopulating hepatocyte RNA with the Fah ${ }^{-1-}$ model involves use of the FAH expression construct to mediate liver repopulation and the GFP-tagged ribosomal protein

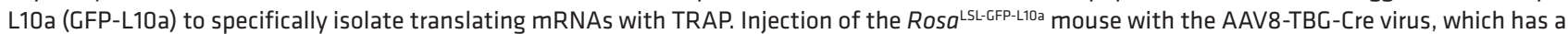
tropism for hepatocytes and has a hepatocyte-specific promoter driving Cre expression in nearly all hepatocytes, allows for immunoprecipitation of translating mRNA from quiescent hepatocytes. (B) Bioanalyzer tracings of affinity-purified RNA from mice treated with or without the TRAP vector. FU, fluorescence units. (C) Representative $(n=3)$ IHC images of GFP show progressive repopulation over time in Fah $/$ - mice as well as complete labeling of

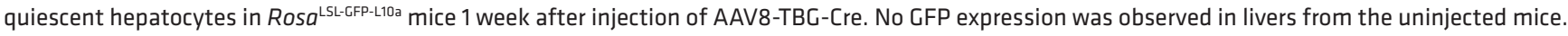
IF of Ki67 and GFP confirmed successful liver repopulation in Fah ${ }^{-/-}$mice injected with the TRAP vector, as all Ki67-positive hepatocytes express GFP. IF costaining also showed global GFP-expressing and rare Ki67-positive hepatocytes, indicating that the control tissue was truly quiescent. Note that a subset of mice showed only partial repopulation at 4 weeks (4-week regeneration after severe injury). Scale bars: $1 \mathrm{~mm}$ (top) and $100 \mu \mathrm{m}$ (bottom).

mutation of Slc7a11 causes a decrease in replicating hepatocytes. These findings indicate the functional significance of $\mathrm{xCT}$ and suggest that activation of Slc7a11 could be used clinically to support therapeutic liver regeneration in the setting of acute liver injury.

\section{Results}

With the goal of specifically isolating repopulating hepatocytes from the injured liver to perform RNA-seq, we initially set out to lineage trace repopulating hepatocytes with GFP and isolate tagged cells by FACS for expression analysis. However, we encoun- tered several problems. First, the fragility of hepatocytes undergoing repopulation led to poor recovery following liver perfusion. Second, the large size of the repopulating hepatocytes hampered the yield and purity of isolated cells by sorting. Finally, the process from organ harvest to cell isolation took more than 2 hours, which may have altered the expression profile.

Next, we turned to TRAP-seq (Figure 1A), which enables the immunoprecipitation of ribosome-bound, translating mRNA from cells that express a fusion protein of the ribosomal protein L10a and GFP (GFP-L10a) (17). The fusion protein was subcloned into the coexpression vector $\mathrm{pKT} 2 / \mathrm{Fah}-\mathrm{mCa} / / \mathrm{SB}$ (15) to construct 
pKT2/Fah-Gfp-L10a//SB (TRAP vector), which expresses FAH together with GFP-L10a. The TRAP vector utilizes the Sleeping Beauty transposon system for stable plasmid integration into the hepatocyte genome (15). The TRAP vector was hydrodynamically injected into $\mathrm{Fah}^{-/}$mice, and NTBC was withdrawn to induce liver injury and create pressure for the selection of hepatocytes that stably express FAH to repopulate the liver. An estimated 0.1\% to $1 \%$ of hepatocytes integrated the plasmid stably into their genomes (20). Tissue was harvested 1 or 4 weeks after injection, and GFPtagged polysomes were extracted to isolate translating mRNAs specifically from repopulating hepatocytes (Figure 1B). No RNA was recovered from mice that were not injected with the TRAP vector, indicating the specificity of TRAP isolation. Three mice in the four-week regeneration group had a greater degree of weight loss (Supplemental Figure 1; supplemental material available online with this article; https://doi.org/10.1172/JCI95120DS1), which was suggestive of more severe injury. Indeed, livers from these mice had large areas lacking GFP staining, indicating a reduced level of initial plasmid uptake (Figure 1C). Hence, we grouped these mice into a separate category termed " 4 -week regeneration after severe injury." Immunofluorescence (IF) analysis of liver sections confirmed that the majority of proliferating hepatocytes also expressed GFP (Figure 1C). Thus, TRAP allows for mRNA isolation selectively from hepatocytes repopulating the injured liver, without contamination from dying hepatocytes or inflammatory cells.

To obtain mRNA from quiescent hepatocytes as a reference for TRAP-seq, we used the Rosa ${ }^{\text {LSL-GFP-L10a }}$ mouse, in which expression of GFP-L10a can be activated following Cre expression (21). We injected Rosa $a^{\text {LSL-GFP-L10a }}$ mice with hepatocyte-specific AAV8TBG-Cre $(22,23)$ and performed TRAP to isolate hepatocyte mRNA 1 week later (Figure 1A). IHC of liver tissue from these mice confirmed that GFP expression was only found in hepatocytes following AAV8-TBG-Cre injection (Figure 1C). GFP and Ki67 colabeling revealed very few actively dividing hepatocytes (Figure 1C), consistent with the quiescent liver state.

High-throughput sequencing of cDNA libraries derived from 16 samples of TRAP-isolated mRNA obtained, on average, 5.8 million uniquely mapped reads (Supplemental Table 1). As expected in pure hepatic mRNA, the 10 most abundant transcripts in the quiescent animals were specific to hepatocytes (Supplemental Table 2) (24-26). Hepatocyte-specific genes such as Alb and Ttr were highly abundant in hepatocytes from all samples, whereas the cholangiocyte markers CK19, CK7, CFTR, and PKD2, as well as transcripts from other cell types in the liver, were nearly undetectable (Supplemental Table 3) $(27,28)$, demonstrating the exquisite specificity of the TRAP method.

Differential gene expression analysis identified 6,745 genes that change in expression in repopulating compared with quiescent hepatocytes (Supplemental Table 4); 3,418 were significantly upregulated and 3,380 downregulated (FDR $\leq 5 \%$ ) (Figure $2 \mathrm{~A}$ ). Hierarchical clustering of the differentially expressed genes showed a distinct separation between quiescent and repopulating hepatocytes (Figure 2B). Notably, the 4-week regeneration group clustered closer to the 1-week regeneration group, rather than to the 4 -week regeneration after severe injury group, demonstrating that TRAP-seq allows identification of different levels of liver regeneration. To establish whether the differentially expressed genes fall into defined regulatory networks, we used pathway analysis and focused on the highly validated Kyoto Encyclopedia of Genes and Genomes (KEGG) network collection $(29,30)$ (Figure 2C). Pathways controlling replication and growth were overrepresented among the upregulated genes, including those regulating the cell cycle and DNA replication, indicating that genes involved in cell replication were activated during liver repopulation, as expected. Strikingly, the GSH metabolic pathway was strongly activated in regenerating hepatocytes, aligning with previous studies showing that control of oxidative stress plays a crucial role in the regenerative response following toxic liver injury (31). Interestingly, metabolic pathways were enriched in both activated and inhibited genes, reflecting the important metabolic regulation of hepatocytes, although the genes at play were different in the 2 groups (Supplemental Table 5). Upregulated metabolic genes included redox processes, whereas repressed genes regulate lipid biosynthesis, corroborating previous findings that hepatocytes limit the activity of metabolic networks to conserve energy for rapid cell replication and DNA synthesis during regeneration (7).

The key regulatory nodes enriched in differentially expressed genes were analyzed with Ingenuity Pathway Analysis, which takes into account the degree of change of each gene to generate putative regulatory networks and predict activation or inhibition of the pathways. We identified 227 upstream regulators, of which 24 met the following additional filters: (a) significant $Z$-scores ( $\geq 2$ for predicted activation and $\leq 2$ for predicted inhibition); (b) at least a 2-fold change in expression; and (c) congruence between the observed fold change and predicted state categories (Table 1). MYC, the most enriched regulator, is a proto-oncogene activated as early as 1 hour after PHx (32) and is also upregulated in liver regeneration induced by carbon tetrachloride and galactosamine (33). Previous work had identified MYC as the strongest driver of liver repopulation in $\mathrm{Fah}^{-/}$mice in a cDNA overexpression screen of more than 40 genes (16), and its overexpression also induces spontaneous hepatocellular carcinoma (HCC) development in the $\mathrm{Fah}^{-/}$mouse model within 8 weeks (34). A second upstream regulator of the proliferative response is the transcription factor FOXM1, which was previously shown to enhance liver repopulation (35). These results indicate that we were indeed able to profile the translating mRNA signature specifically in repopulating hepatocytes and demonstrate that TRAP-seq is a robust methodology for identifying enriched pathways and upstream regulators.

Next, we set out to compare the transcriptional changes of regenerating hepatocytes in $\mathrm{Fah}^{-/-}$mice recovering from toxic injury with those occurring following $\mathrm{PHx}$, a paradigm of noninjury regeneration. First, we reanalyzed previous RNA-seq data from whole-liver homogenates after $\mathrm{PHx}$ (36) and identified 2,321 differentially expressed genes, 1,449 of which were activated and 872 inhibited (Figure 2D). Hierarchical clustering showed a distinct separation of gene regulation at various time points after PHx (Figure 2E). Interestingly, gene expression at 1 hour clustered closer with quiescent hepatocytes, indicating that at this very early time point only a few early-response genes were transcriptionally regulated. Pathway analysis $(29,30)$ showed enrichment of genes regulating cell-cycle and DNA synthesis 
A

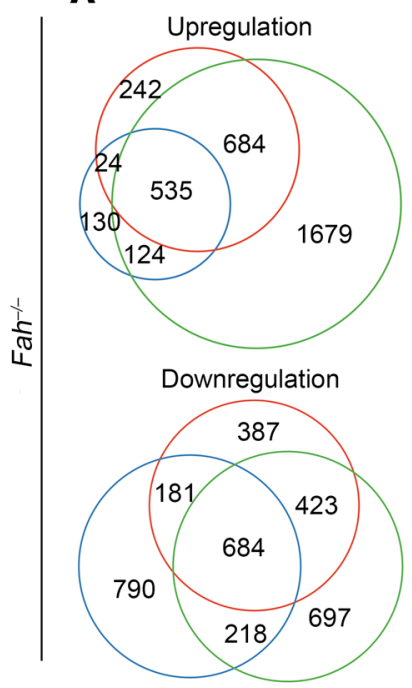

D

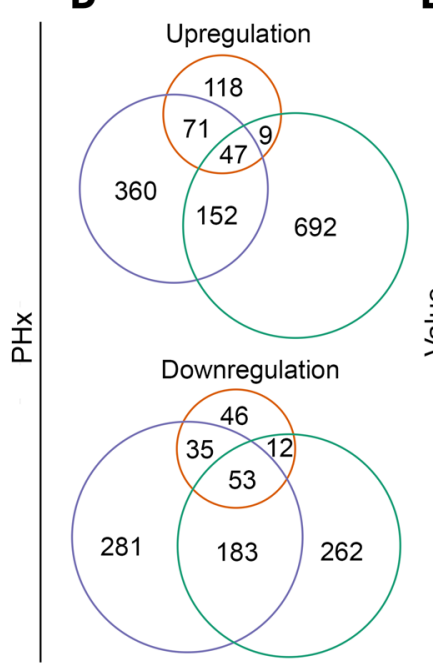

B

$\square$ Quiescent
$\square$ 1-week regeneration

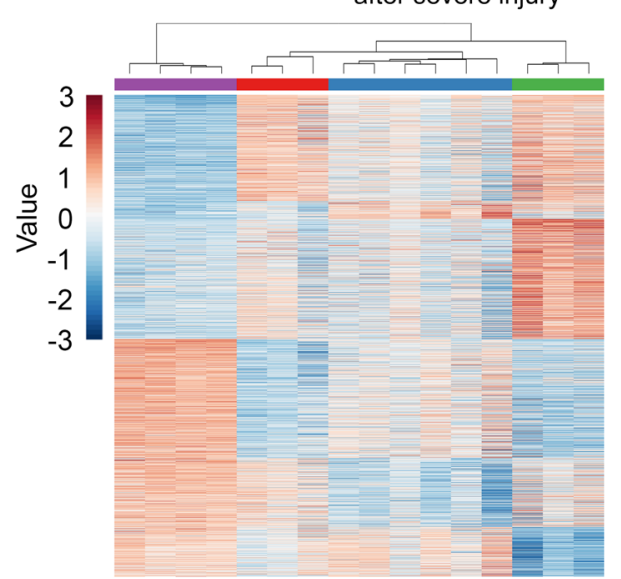

C

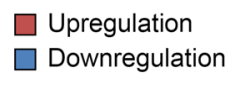

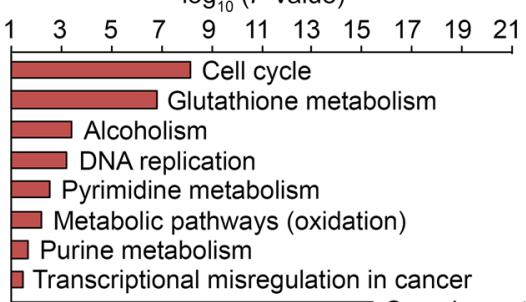

Complement and coagulation Steroid hormone biosynthesis

\begin{tabular}{l}
\hline Steroid horm \\
\hline Metabolic pathways (lipid)
\end{tabular}

$\square$ Bile secretion

Retinol metabolism

Chemical carcinogenesis

Drug metabolism

Lysosome

\section{$\mathbf{F}$}

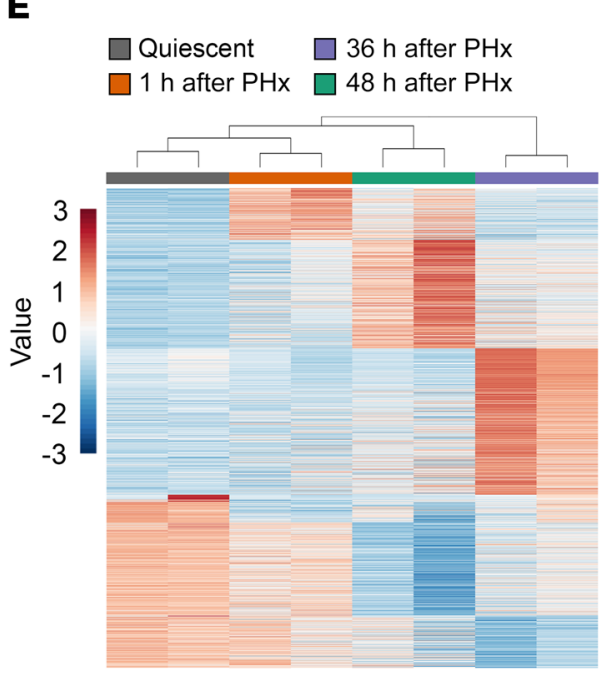

$\square$ Upregulation

Downregulation

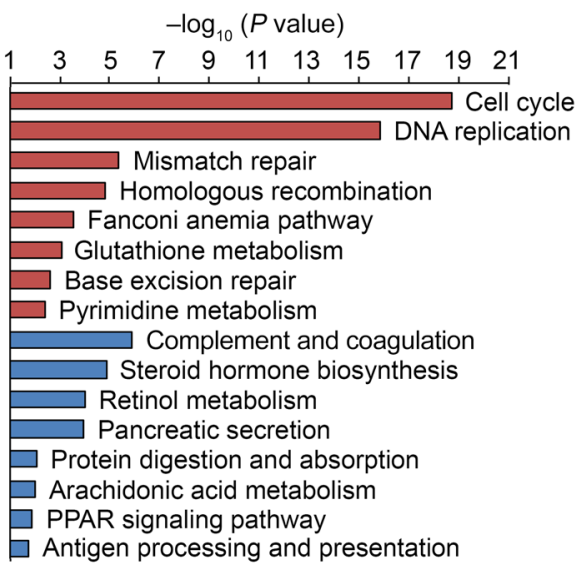

Figure 2. TRAP-seq identifies differentially expressed genes specific to repopulating hepatocytes in the Fah -/- $^{-}$model. (A and D) Differential expression analysis identified 6,745 (3,418 upregulated and 3,380 downregulated) and 2,321 (1,449 upregulated and 872 downregulated) genes as being significantly altered in repopulating hepatocytes in the $\mathrm{Fah}^{-/-}$(A) and PHx (D) models (36), respectively, compared with quiescent controls. Red, 1-week Fah ${ }^{-/-}$regeneration and 1 hour after PHx; blue, 4-week Fah ${ }^{-/-}$regeneration and 36 hours after PHx; green, 4-week Fah ${ }^{-/-}$regeneration after severe injury and 48 hours after PHx. (B and E) Hierarchical clustering of differentially expressed genes of quiescent and repopulating hepatocytes at different time points. (C and F) KEGG pathways significantly enriched for the sets of activated and repressed genes, respectively, in the $\mathrm{Fah}^{-/-}$(C) and PHx (F) data sets.

pathways among the upregulated genes and those regulating immune and metabolic pathways among the downregulated genes (Figure 2F).

We compared the gene expression changes between the $\mathrm{Fah}^{-1}$ and PHx models, defining congruent genes as those regulated in the same direction in both models for at least 1 time point. We identified a total of 1,236 congruent genes, 790 of which were activated and 446 repressed (Figure 3A). Gene expression changes that occurred at all time points in the $\mathrm{Fah}^{-/-}$repopulation mice were most similar to the changes observed in the PHx model at later time points (36 or 48 hours after PHx), as shown by the high percentage of congruence. Additionally, we found that the percentage of congruence was higher among the upregulated genes, indicating a more similar gene activation pattern in the
2 regeneration models. We discovered that the top upregulated congruent genes - ranked by mean fold change in $\mathrm{Fah}^{-/-}$mice and subsequently retrieved from the PHx data set - were associated with GSH metabolism, including the genes Slc7a11 and Gsta1 (Supplemental Table 6) (18). This was confirmed by pathway analysis, in which GSH metabolism was highly enriched in the congruently upregulated genes, along with cell-cycle, DNA replication, and DNA repair pathways (Figure 3C). Immune response and metabolic pathways were enriched among the congruently downregulated genes (Figure 3D). Interestingly, the majority of the congruent genes did not show a significant change 1 hour after PHx (Supplemental Table 6), as at this stage, hepatocytes still resembled quiescent hepatocytes, with activation of only a few immediate early genes (2). 
Table 1. Upstream regulators predicted by Ingenuity Pathway Analysis

\begin{tabular}{|c|c|c|c|c|c|}
\hline $\begin{array}{l}\text { Upstream } \\
\text { regulator }\end{array}$ & $\begin{array}{l}\text { Fold } \\
\text { change }\end{array}$ & Molecule type & $\begin{array}{l}\text { Predicted } \\
\text { state }\end{array}$ & Z-score & $P$ value \\
\hline MYC & 3.08 & Transcription regulator & Activated & 4.73 & $1.15 \times 10^{-24}$ \\
\hline SREBF1 & 0.26 & Transcription regulator & Inhibited & -3.97 & $3.32 \times 10^{-16}$ \\
\hline THRB & 0.50 & Nuclear receptor & Inhibited & -2.28 & $4.26 \times 10^{-10}$ \\
\hline E2F1 & 2.53 & Transcription regulator & Activated & 2.59 & $2.06 \times 10^{-9}$ \\
\hline FOXM1 & 15.67 & Transcription regulator & Activated & 3.23 & $4.79 \times 10^{-9}$ \\
\hline EGR1 & 0.33 & Transcription regulator & Inhibited & -2.09 & $4.33 \times 10^{-8}$ \\
\hline HBB-B1 & 0.11 & Transporter & Inhibited & -2.07 & $7.41 \times 10^{-8}$ \\
\hline SPARC & 0.30 & Other & Inhibited & -3.62 & $1.43 \times 10^{-7}$ \\
\hline CSF1 & 0.48 & Cytokine & Inhibited & -2.95 & $2.63 \times 10^{-7}$ \\
\hline HBB-B2 & 0.11 & Other & Inhibited & -2.68 & $5.31 \times 10^{-7}$ \\
\hline E2F2 & 4.85 & Transcription regulator & Activated & 2.75 & $9.10 \times 10^{-7}$ \\
\hline USF2 & 0.35 & Transcription regulator & Inhibited & -2.49 & $2.40 \times 10^{-6}$ \\
\hline AGTR1 & 0.40 & GPCR & Inhibited & -2.70 & $6.04 \times 10^{-6}$ \\
\hline LMNB1 & 6.69 & Other & Activated & 2.56 & $6.26 \times 10^{-6}$ \\
\hline CCNE1 & 3.70 & Transcription regulator & Activated & 2.07 & $2.14 \times 10^{-5}$ \\
\hline MLXIPL & 0.40 & Transcription regulator & Inhibited & -3.70 & $3.19 \times 10^{-5}$ \\
\hline TFEB & 0.35 & Transcription regulator & Inhibited & -2.05 & $3.33 \times 10^{-4}$ \\
\hline S100A6 & 2.92 & Transporter & Activated & 2.85 & $1.20 \times 10^{-3}$ \\
\hline CTGF & 0.44 & Growth factor & Inhibited & -2.04 & $1.26 \times 10^{-3}$ \\
\hline TAS1R3 & 0.42 & GPCR & Inhibited & -2.14 & $2.02 \times 10^{-3}$ \\
\hline IL15 & 0.37 & Cytokine & Inhibited & -2.70 & $3.28 \times 10^{-3}$ \\
\hline FASN & 0.14 & Enzyme & Inhibited & -2.11 & $1.77 \times 10^{-2}$ \\
\hline TNK1 & 0.46 & Kinase & Inhibited & -2.83 & $2.03 \times 10^{-2}$ \\
\hline MLYCD & 0.50 & Enzyme & Inhibited & -2.00 & $2.06 \times 10^{-2}$ \\
\hline
\end{tabular}

Filter criteria: (a) significant Z-scores ( $\geq 2$ for predicted activation and $\leq 2$ for predicted inhibition); (b) at least 2-fold change in expression; and (c) congruence between the observed fold change and predicted state categories.

Of note, 2 of the top congruently upregulated genes, $L y 6 d$ and $P b k$, are not typically expressed in hepatocytes. The average fragments per kilobase of transcript per million mapped (FPKM) reads for these genes in the quiescent hepatocytes were 2.7 and 0.01 , but increased to 504.4 and 6.6 in regenerating hepatocytes, respectively (Supplemental Table 4). Ly6d expression has been shown to be associated with HCC and liver regeneration after injury (37, 38), while $P b k$ has been detected in HCC and cholangiocarcinoma $(39,40)$. This further demonstrates the sensitivity and specificity of TRAP-seq in detecting expression changes in a unique subpopulation of the liver - that of the regenerating hepatocytes.

Additionally, we identified genes that were only changed in 1 model but not the other (unique genes), of which 5,510 were unique to $\mathrm{Fah}^{-1-}$ mice, and 1,033 were unique to the PHx model (Figure 3B and Supplemental Table 5). Of note, in both models, the percentage of unique genes compared with the total number of differentially expressed genes was approximately $81 \%$. However, in the $\mathrm{Fah}^{-1-}$ mice, up- and downregulated genes each constituted $50 \%$ of the unique genes, whereas in the PHx model, the upregulated and downregulated genes made up $64 \%$ and $36 \%$ of the unique genes, respectively. To further identify the biological pathways specific to each model, pathway enrichment analysis was performed on the unique genes $(29,30)$, and overrepresented networks were identified (Figure 3, C and D). In $\mathrm{Fah}^{-1-}$ mice, liver injury response categories such as alcoholism and viral carcinogenesis were uniquely activated, while immune response and metabolic pathways were uniquely inhibited. On the other hand, no significant pathway activation was unique to the PHx model, whereas the pancreatic secretion and protein and fat digestion/ absorption pathways were uniquely inhibited. The striking difference in enriched pathways demonstrates the gene expression signatures that differentiate the 2 regeneration paradigms, in which injury response and immune modulation are unique to $\mathrm{Fah}^{-/}$mice and nutrient redistribution is integral to the PHx model.

Recently, single-molecule RNA-FISH combined with singlecell RNA-seq (scRNA-seq) has been applied to reconstruct the spatial heterogeneity and identify novel zonal signature genes within the quiescent liver (41). While TRAP-seq utilizes bulk RNA-seq and therefore cannot inform a spatial resolution of transcriptional changes during regeneration, we compared the expression profiles of quiescent hepatocytes from TRAP-seq with the scRNA-seq data. We reasoned that, since all hepatocytes express GFP-L10a in the quiescent liver (Figure 1), the isolated transcripts from TRAPseq should have an equal representation of the genes identified from the 9 different subpopulations by scRNA-seq. As expected, we found significant overlap between TRAP-seq and all 9 layers of scRNA-seq, with an average of 10,405 common genes, constituting $90.7 \%$ of the genes detected by TRAP-seq (Supplemental Figure 2). Thus, TRAP-seq enables unbiased RNA isolation from all layers of hepatocytes.

The comparison of the $\mathrm{Fah}^{-/}$and PHx models revealed Slc7a11 as the most significantly activated gene in both paradigms, with a remarkable increase of 900 -fold in the former and 200 -fold in the latter (Supplemental Table 5). Slc7a11 encodes xCT, a sodium-independent transporter for cystine import and glutamate export (19). After entering the cell, cystine is rapidly reduced to cysteine, a precursor for GSH synthesis necessary for cellular defense against oxidative stress (18). Previous studies indicated that deficiency of glutamate cysteine ligase, the rate-limiting enzyme in GSH synthesis, leads to decreased hepatocyte proliferation in vitro and delayed regeneration after PHx $(42,43)$. However, the role of $\mathrm{xCT}$ in liver regeneration has not been studied. We hypothesized that $\mathrm{xCT}$ upregulation supports actively repopulating hepatocytes to defend against increased oxidative stress during injury and regeneration (Figure $4 \mathrm{~A}$ ).

To evaluate the role of $\mathrm{xCT}$ in liver regeneration, we first validated our observations from RNA-seq with quantitative real-time reverse transcription PCR (qRT-PCR) on TRAP-purified mRNA and confirmed a significant upregulation of Slc7a11 and Gsta1 transcripts in repopulating hepatocytes (Supplemental Figure 3A). Western blot analysis showed an increase in $\mathrm{xCT}$ protein in repopulating livers (Supplemental Figure 3B). Of note, there was low $\mathrm{xCT}$ expression in the quiescent liver, albeit no mRNA transcripts were present in hepatocytes. One possibility is that whole-liver homogenate was used for the protein analysis, and thus xCT protein from other cell types such as macrophages were detected (19). Alternatively, the protein stability of Slc7a11 could exceed its RNA turnover rate. Regardless, expression of Slc7a11 was significantly activated in the regenerating liver. 

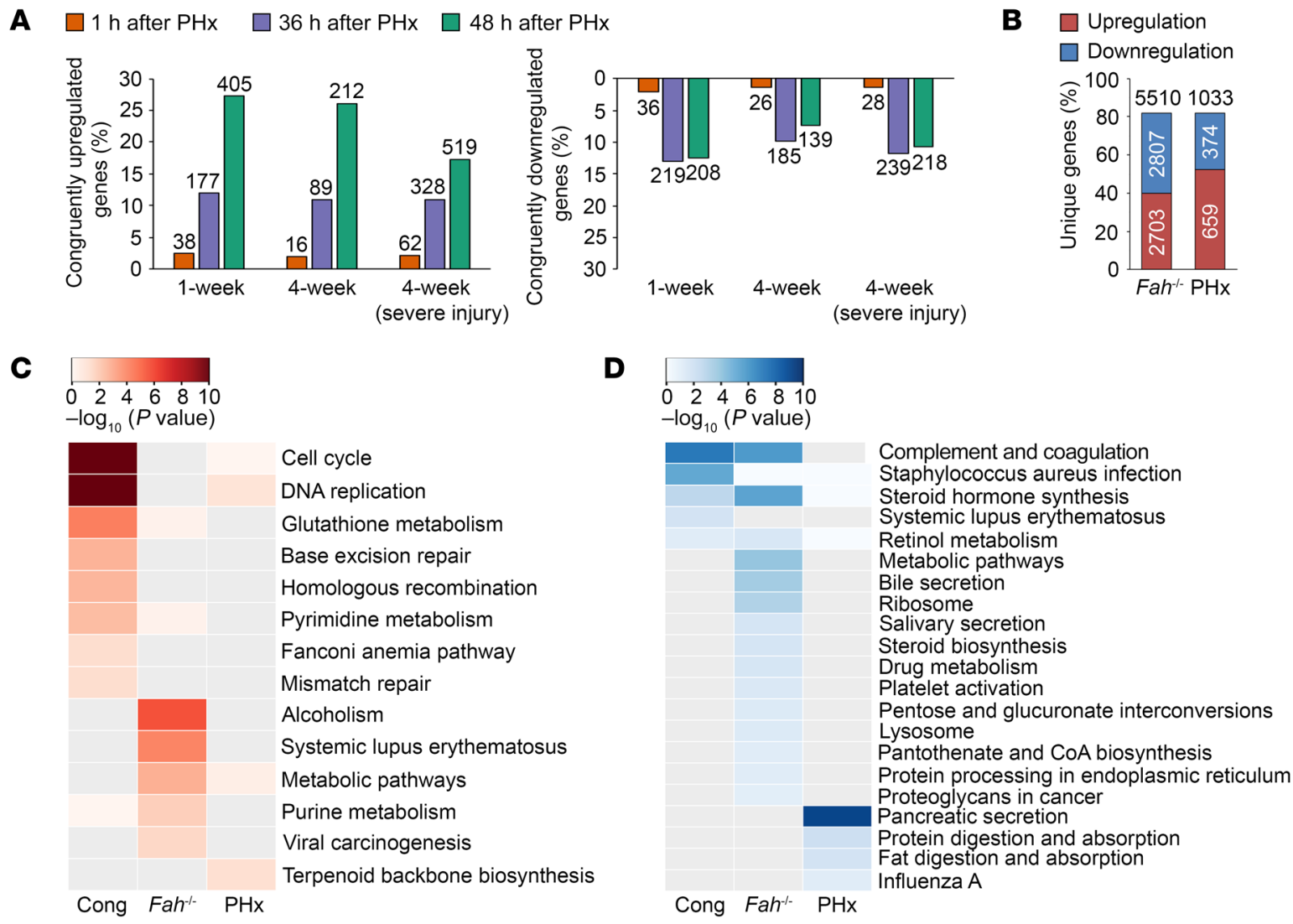

Figure 3. Comparison of the $\mathrm{Fah}^{-/-}$TRAP-seq data with RNA-seq data from the PHx model identifies common and unique characteristics of liver repopulation paradigms. (A) A total of 1,236 genes were significantly altered in the same direction in both models (36) for at least 1 time point (congruent genes). Of these genes, 790 were activated and 446 inhibited. Labels indicate the number of congruent genes at each time point. (B) A total of 5,510 and 1,033 genes were uniquely changed in the $\mathrm{Fah}^{-/-}$and $\mathrm{PHx}$ models, respectively. (C and D) Comparison of the KEGG pathways enriched for genes upregulated (C) and downregulated (D) in the congruent (Cong) and unique gene sets.

We next sought to investigate whether oxidative stress is increased in $\mathrm{Fah}^{-/}$livers during regeneration. We used immunohistochemical methods to detect markers of lipid peroxidation (malondialdehyde and 4-hydroxynonenal) and protein nitration (nitrotyrosine). We observed an accumulation of redox metabolites in the injured livers compared with healthy, quiescent livers (Supplemental Figure 3C). These results indicate that Slc7a11 mRNA expression and xCT protein levels are highly enriched in repopulating hepatocytes in the presence of increased reactive oxygen and nitrogen species, suggesting a functional role of $S l c 7 a 11$ in the regulation of liver regeneration.

To examine the functional importance of xCT activation in regenerating hepatocytes, we constructed plasmids coexpressing Fah and overexpressing Slc7a11 (Fah-Slc7a11) or Gfp (Fah-Gfp). We performed a competition assay, in which equimolar amounts of Fah-Gfp and Fah-Slc7a11 were injected into $\mathrm{Fah}^{-/-}$mice, followed by NTBC withdrawal (Figure 4B). After 4 weeks of repopulation, we observed a 2.5-fold enrichment of Fah-Slc7a11 plasmid relative to the Fah-Gfp control plasmid by qPCR of extracted liver genomic DNA (Figure 4C) as well as overrepresentation of HA-tagged, xCT-expressing hepatocytes compared with GFP-expressing cells (Figure 4D). These results demonstrate a positive selection for hepatocytes overexpressing xCT, even above the already striking activation of endogenous Slc7a11.
To test whether Slc7a11 is required for liver regeneration, we used CRISPR/Cas9 to inactivate $S l c 7 a 11$ specifically in the repopulating hepatocytes. We coexpressed FAH with either 10 single-guide RNAs (sgRNAs) targeting Slc7a11 exons (Fah-sgSlc7a11) or 10 control sgRNAs targeting luciferase (Fah-gCtl) and performed hydrodynamic tail-vein injection of these sgRNAs, together with adeno-associated virus 8 (AAV8) expressing Staphylococcus aureus Cas9 (SaCas9) to allow for hepatocyte-specific expression of the SaCas9 nuclease (44), which efficiently introduces indels comparable to those of Cas9 from S.pyogenes (45) (Figure 4E). Liver repopulation was then carried out for 4 weeks. To quantify and characterize the mutations induced by CRISPR/Cas9, we extracted genomic DNA from the repopulating livers, PCR amplified exon 1 for Sanger sequencing, and performed tracking of indels by decomposition (TIDE) analysis (46). We found that the 2 sgRNAs targeting the first exon of Slc7a11 exhibited different mutation efficiency: $29.5 \%$ and 51.6\%, respectively (Supplemental Figure 4, A and B). Furthermore, the main mutation introduced by SaCas9 in either sgRNA was a 5-nucleotide deletion, with an efficacy of $27.9 \%$ and $51.6 \%$, respectively. The difference in mutation rate could be due to the slight difference in the protospacer-associated motif (PAM) sequence (NNGRRT) of the 2 sgRNAs, CTGAGT and AAGGGT (45). Nonetheless, TIDE analysis demonstrated that Slc7a11 was mutated through the expression of SaCas9 in the hepatocytes. 
A

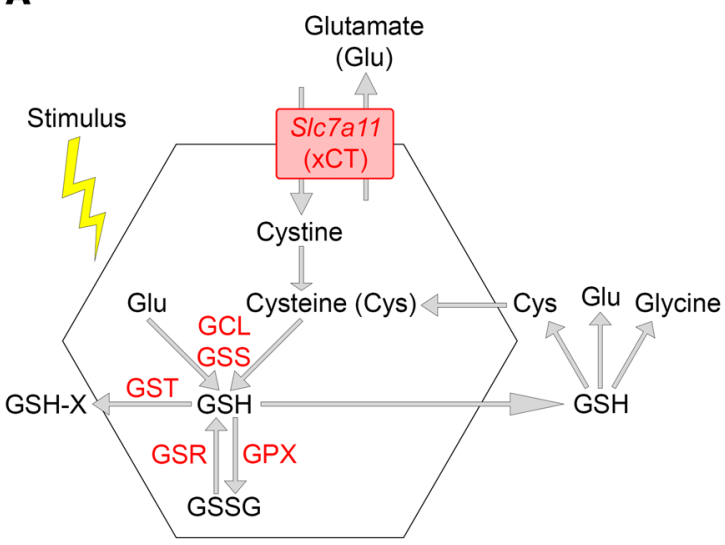

Repopulating hepatocyte

E
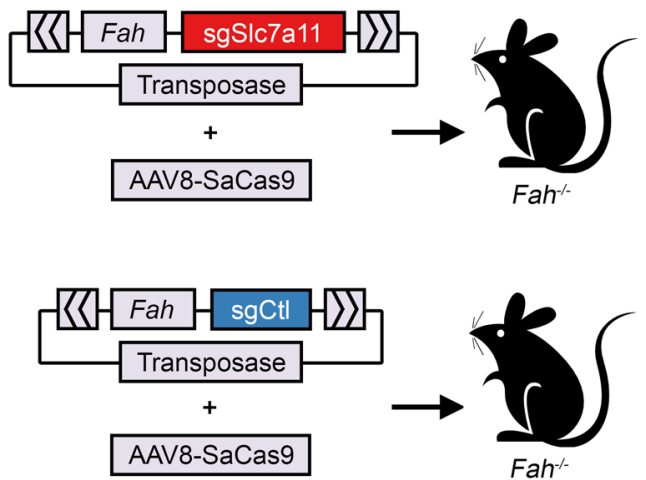

B

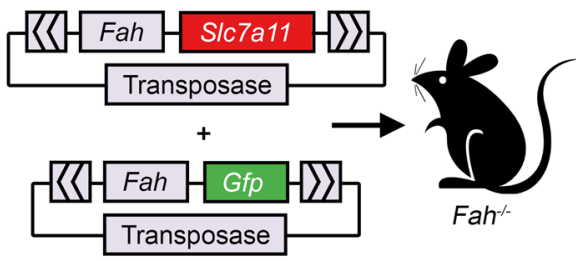

C

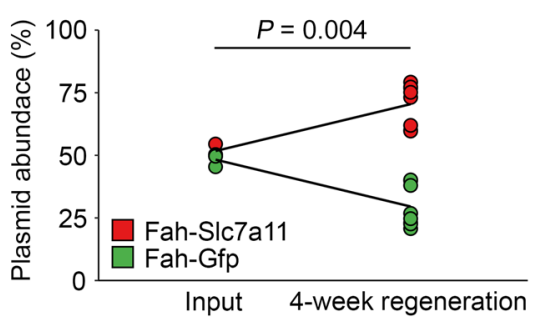

$\mathbf{F}$
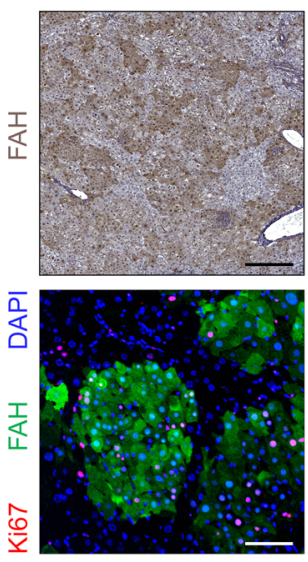

$\mathrm{sgCtl}$
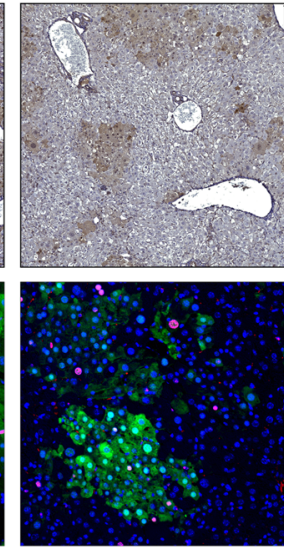

sgSIc7a11
D
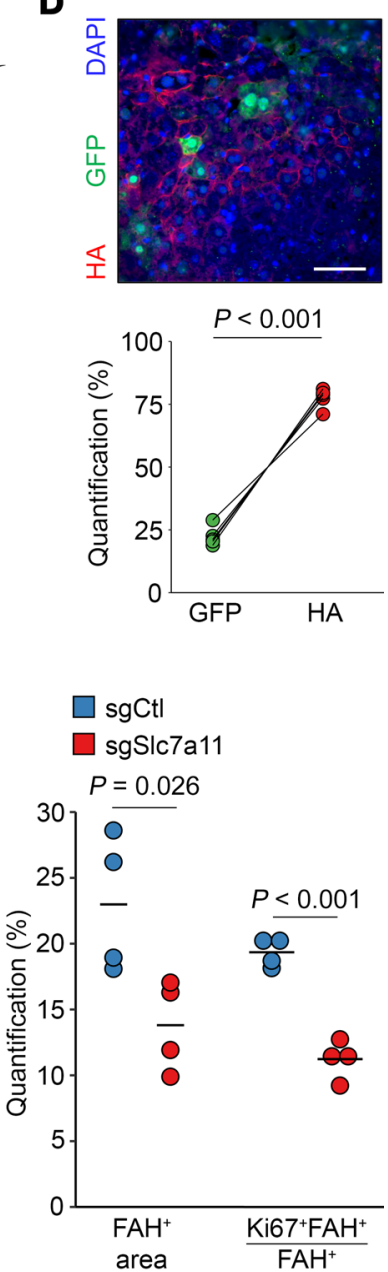

Figure 4. SIc7a11 enhances hepatocyte repopulation. (A) The SIc7a11 gene product (xCT) imports cystine, which is used for GSH synthesis to alleviate oxidative stress. Several GSH metabolic enzymes were significantly (FDR $\leq 5 \%$ ) upregulated (red) in repopulating hepatocytes from Fah ${ }^{-/-}$mice. GSSG, glutathione disulfide; GCL, glutamate-cysteine ligase; GSS, glutathione synthetase; GST, glutathione S-transferase; GSR, glutathione reductase; GPX, glutathione peroxidase. (B) Schematic of the competition assay to determine the effects of Slc7a11 overexpression on repopulation. (C) The Fah-SIc7a11 plasmid was significantly enriched after 4 weeks of repopulation. A 1-sample, 2-tailed Student's $t$ test was used to compare the ratio of 2 plasmids before and after repopulation $(n=8)$. (D) Representative IF staining and quantification showing a significant increase in xCT-positive hepatocytes. A paired, 2 -tailed Student's $t$ test was used to compare HA- and GFP-expressing hepatocytes $(n=5)$. Scale bar: $100 \mu \mathrm{m}$. (E) Schematic of the CRISPR/Cas9 system used to inactivate SIc7a11 in Fah/- mice. sgCtl, sgRNAs targeting firefly luciferase. (F) Representative IHC and IF images and quantification showing a significant reduction in repopulation nodules and replicating hepatocytes in mice treated with sgRNAs targeting S/c7a11 (sgSIc7a11) compared with control mice treated with sgCtl. A 2-sample, 2-tailed Student's $t$ test was used to compare groups ( $n=4$ each). Scale bars: $300 \mu \mathrm{m}$ (top) and $100 \mu \mathrm{m}$ (bottom).

We measured weight changes over the 4-week period of liver repopulation and found no significant weight differences in mice treated with Slc7a11 sgRNAs compared with those treated with control sgRNAs (Supplemental Figure 4C). Likewise, we detected no significant difference in the liver weight to body weight ratio by the end of the 4-week period (Supplemental Figure 4D). However, sgSlc7a11-treated mice had smaller FAH repopulation nodules and fewer Ki67/FAH double-positive hepatocytes compared with sgCtl-treated mice (Figure 4F), indicating that Slc7a11 mutation inhibits replication of FAH-expressing cells during liver injury. It should be noted that these results are probably an underrepresentation of the true effect of Slc7a11 mutation, as only hepatocytes homozygous, not those that are heterozygous, for inactivation of Slc7a11 are expected to be at a growth disadvantage. Furthermore, redundant pathways could compensate for the loss of Slc7a11 (47).
Together, these studies demonstrate the functional importance of xCT during liver repopulation and show that Slc7a11 overexpression is sufficient to accelerate repopulation, whereas Slc7a11 inactivation, while not completely abrogating regeneration, hinders hepatocyte replication.

Finally, we investigated the mechanism of xCT activation during liver repopulation. Several transcription factors have been shown to regulate Slc7a11 expression in different contexts: nuclear factor E2-related factor 2 (NRF2) activates xCT during redox stress (48), activating transcription factor 4 (ATF4) upregulates xCT under ER stress (49), octamer-binding transcription factor (OCT1) disinhibits Slc7a11 following ethanol exposure (50), and p53 inhibits XCT under normal tumor suppression conditions (51). Additionally, ATF4 is suggested to regulate the basal levels of Slc7a11 expression (49). 
A

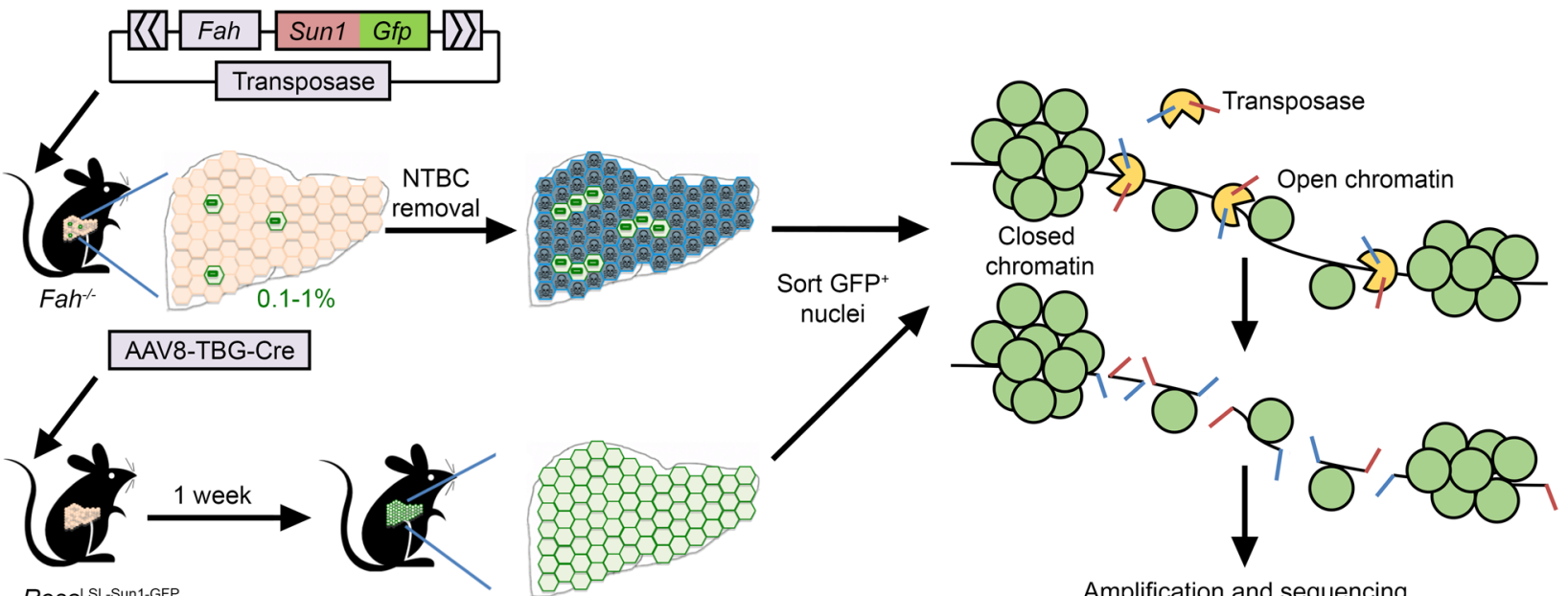

Rosa ${ }^{\text {LSL-SUn1-GFP }}$

Amplification and sequencing

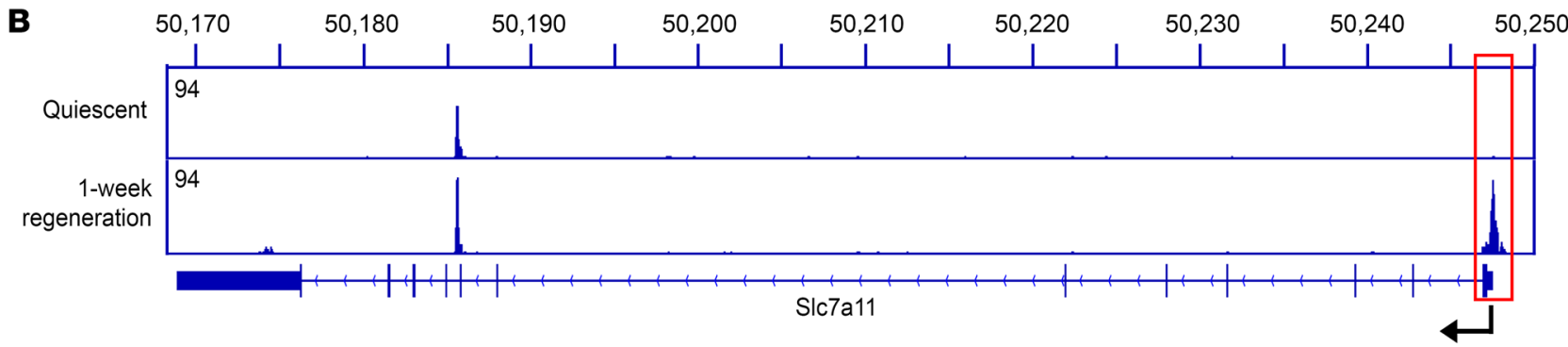

C

NRF2

TGCATGATGATGCAAATTCTCCAGGTTTGCATCAGCC

ATF4

ATF4(-)
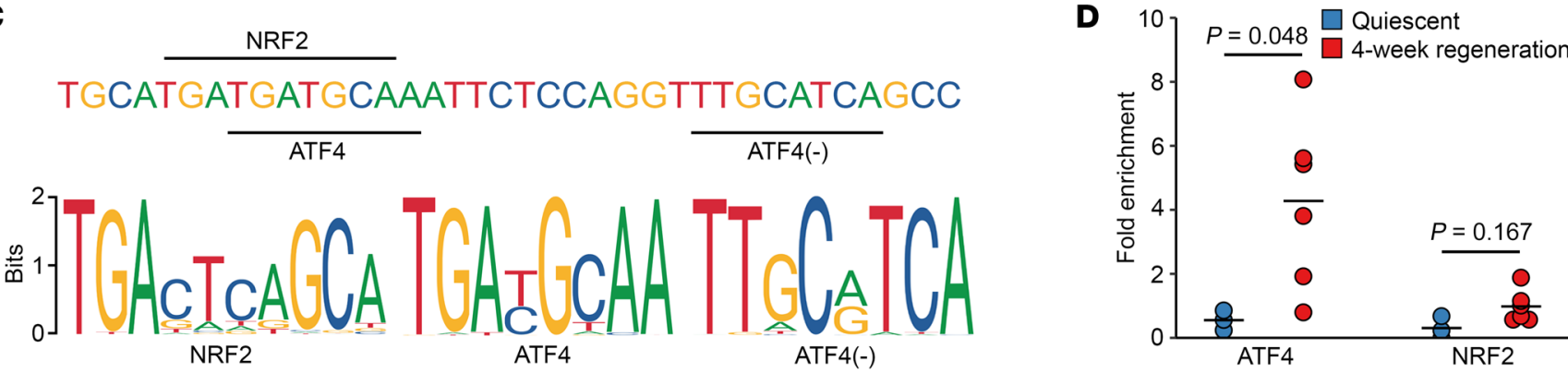

Figure 5. SIc7a11 is activated by ATF4 during liver repopulation. (A) Schematic of our approach utilizing the GFP-labeled nuclear envelope protein SUN1 to isolate hepatocyte nuclei $(53)$, followed by ATAC-seq $(54,55)$ analysis. (B) ATAC-seq identified an open chromatin state at the promoter region of SIc7a11 specifically in regenerating hepatocytes ( $n=2$, quiescent; $n=4$, 1-week regeneration). (C) The open chromatin region of the SIc7a11 promoter contains binding motifs for NRF2 and ATF4. (D) ChIP-qPCR showed a 4-fold enrichment of ATF4 binding to the Slc7a11 promoter after 4 weeks of liver regeneration, while no enrichment in NRF2 binding was observed. The Wilcoxon rank-sum test was used to compare the differential binding in regenerating and quiescent livers ( $n=3$, quiescent; $n=6$, 4-week regeneration).

We first performed unbiased chromatin accessibility profiling to identify regulatory elements at the Slc7a11 locus in hepatocytes in the basal and repopulating state. We used the isolation of nuclei tagged in specific cell types (INTACT) system to label the nuclei of regenerating hepatocytes (52). Specifically, the nuclear envelope protein SUN1 was tagged with GFP (53), and the resulting fragment was subcloned into the FAH coexpression construct (FahSun1-Gfp). One week after the $\mathrm{Fah}^{-/}$mice were repopulated with Fah-Sun1-Gfp, livers were harvested and sorted for GFP-positive nuclei (Figure 5A). As a quiescent control, we injected $R$ os $^{\text {LSL-Sunl-GFP }}$ mice with AAV8-TBG-Cre and sorted hepatocytes after 1 week (Figure 5A). We used the assay for transposase accessible chromatin using sequencing $($ ATAC-seq) $(54,55)$ to profile the chromatin landscape changes after 1 week of regeneration. Remarkably, the Slc7a11 promoter is highly accessible in the regenerating hepatocytes, as indicated by the strong peak present 1 week after regeneration (Figure 5B). In comparison, we observed no peak at the promoter in the quiescent liver, demonstrating a heterochromatic state in healthy liver cells. This observation coincides with our TRAP-seq analysis, in which no Slc7a11 transcripts were detected in quiescent hepatocytes, but became highly abundant in regenerating hepatocytes (Supplemental Figure 3A).

Next, to determine how Slc7a11 is activated, we performed a motif search at the open chromatin region of the activated promoter and identified a potential NRF2-binding site 39 bases and 2 potential ATF4-binding sites 39 and 66 bases upstream 
of the transcriptional start site (Figure 5C). To assess whether ATF 4 or NRF2 binds to the Slc7a11 promoter during liver repopulation, we carried out ChIP-qPCR in quiescent and 4-week regenerating livers. We detected a significant 4 -fold enrichment of bound ATF 4 at the Slc7a11 promoter in regenerating hepatocytes relative to that seen in quiescent controls. In contrast, NRF2 binding was undetected in either condition (Figure 5D), suggesting that ATF4, but not NRF2, activates Slc7a11 transcription during liver repopulation.

\section{Discussion}

Here, we performed what we believe to be the first expression profile specific to repopulating hepatocytes by integrating the TRAP assay with the $\mathrm{Fah}^{-/-}$mouse model. We identified important signaling networks and regulators, including upregulation of the cell-cycle and GSH metabolic pathways, and several activated transcription factors such as MYC and FOXM1. Bioinformatics analysis comparing the gene expression of $\mathrm{Fah}^{-/-}$and $\mathrm{PHx}$ regeneration models identified pathways common to both models, i.e., cell cycle and GSH metabolism pathway genes among the congruently activated genes, and immune response pathway genes among the congruently inhibited genes. We also observed that liver damage pathways are uniquely upregulated in $\mathrm{Fah}^{-/}$mice, while altered biosynthetic activity is a main theme in the PHx model.

A recent study utilizing single-cell technology to reconstruct the spatial heterogeneity of the liver had identified 9 distinct layers of gene expression profiles in quiescent hepatocytes (41). We showed that transcripts identified from TRAP-seq significantly overlapped with those found in scRNA-seq, regardless of the layer, demonstrating the sensitivity and specificity of TRAP-seq in isolating transcripts from pure hepatocytes. Nonetheless, there are several differences between the 2 techniques. First, TRAP-seq utilizes bulk RNA-seq and therefore could not capture the zonal information by scRNA-seq. Second, TRAP-seq isolates mRNA bound to the ribosomal subunit L10a and hence only captures the actively translating mRNA. Third, TRAP-seq does not require cell sorting and therefore bypasses the time-consuming sample preprocessing required for scRNA-seq. Previous efforts to isolate intact regenerating hepatocytes after hydrodynamic injection have been unsuccessful, rendering TRAP-seq a valuable alternative. Future work could apply cell layer-specific expression of GFP-L10a to shed light on the zonal responses to liver injury and regeneration.

Previous work has demonstrated the importance of controlling oxidative stress during liver regeneration to allow hepatocyte replication, as elevation of ROS induces a compensatory upregulation of GSH to inhibit irreversible cell damage and promote hepatic replication (56). In support of the central role of GSH in liver regeneration, inhibition or deficiency of glutamate cysteine ligase, the rate-limiting enzyme in GSH synthesis, leads to downregulation of cyclin expression, decreased hepatocyte proliferation in vitro, and delayed regeneration after PHx $(42,43)$. Furthermore, GSH is depleted in acetaminophen-induced liver injury by the toxic metabolite NAPQI (57), pointing to the importance of GSH detoxification and ROS homeostasis in various regenerative paradigms.

Importantly, our results indicate that $S l c 7 a 11$ becomes dramatically activated in repopulating hepatocytes, and we further show that ectopic expression of XCT concomitantly with the onset of liv- er injury promotes regeneration, probably by shielding repopulating hepatocytes from oxidative stress. These results highlight the therapeutic potential of activating $S l c 7 a 11$ as a treatment for acute liver injury. We did not observe any health complications in mice overexpressing Slc7a11 during the 4-week period of repopulation in the $\mathrm{Fah}^{-/}$mouse. However, determining whether this approach is beneficial in managing chronic liver injury and whether longterm xCT activation is safe will require further examination.

Recent studies have found Slc7a11 to be highly expressed in HCC, breast cancer cells, and gastrointestinal tumors (58-60) and have shown that pharmacological $\mathrm{xCT}$ inhibition induces growth arrest in cancer cells and decreases tumor size in mouse models (59, $60)$. Therefore, it is possible that regenerating hepatocytes experience metabolic requirements similar to those seen in cancer cells to increase GSH availability. This raises the question of the safety of using $\mathrm{xCT}$ antagonists in patients with HCC, as both the growth of cancer cells and regenerating hepatocytes would be inhibited. Interestingly, in our gene inactivation studies, while a decrease in replicating hepatocytes during regeneration was observed, Slc7a11 inhibition did not completely abrogate liver repopulation, and the mice treated with sgRNAs against Slc7a11 were still able to restore full body weight after 4 weeks of regeneration. This observation is consistent with recent findings that $\mathrm{xCT}$ deficiency alone is not sufficient to induce liver injury but exacerbates injury when combined with a secondary stress such as a high-iron diet (61) or inhibition of the transsulfuration pathway (62). In addition, as discussed above, Slc7a11 was probably not inactivated for both alleles in all regenerating hepatocyte clones. Furthermore, genetic redundancy has been proposed to underlie liver regeneration, as loss of any single gene rarely leads to complete inhibition of the regenerative process (3).

In conclusion, this study demonstrates the feasibility of TRAPseq for cell type-specific mRNA isolation of hepatocytes and identifies Slc7a11 as a driver that promotes recovery after acute liver injury. Likewise, TRAP could be used to label other cell types in the liver to study their roles in acute liver injury. For instance, by combining the Rosa ${ }^{\text {LSL-GFP-L10a }}$ mouse with a biliary-specific Cre or stellate cellspecific Cre transgene, it will be possible to profile the cell type-specific gene expression for these cells during injury and regeneration.

\section{Methods}

The primer sequences are listed in Supplemental Table 7.

Plasmid construction. The plasmid C2-EGFP-L10a was provided by Nathaniel Heintz (The Rockefeller University, New York, NY, USA). The GFP-L10a coding sequence was amplified by PCR using the primers L10a-R-BsiWI and MfeI-EGFP-F and subcloned into the vector $\mathrm{pKT} 2 / \mathrm{Fah}-\mathrm{mCa} / / \mathrm{SB}$ (15) at the EcoRI and BsiWI restriction sites. The vector utilizes the Sleeping Beauty (SB) transposon system to enable integration of transgene sequences into the genome. The Slc7a11 cDNA was purchased (MG225346, OriGene) and amplified by PCR with the primers Slc7a11_clone_F1 and Slc7a11_psmd_bcd-R or Slc7a11-HA_bcd-R to include the HA tag. For the CRISPR/Cas9 studies, the vector pKT2/Fah-SpCas9//SB (34) was used to replace the SpCas9 with the SaCas9 sgRNA scaffold and introduce the subcloning site for further sgRNA subcloning using the oligonucleotides SaCas9Ins-F and - R and the restriction enzymes SapI and EcoRI to generate the vector pKT2/Fah-SaCas9//SB. Next, 10 sgRNAs targeting the exon regions of Slc7a11 were designed with the online CRISPR RGEN Tools 
(63) and DESKGEN Cloud (64). Ten sgRNAs against luciferase were designated as the control, and the oligonucleotides were subcloned into the pKT2/Fah-SaCas9//SB vector at the SapI restriction sites. For the ATAC-seq study, SUN1-GFP fragments with EcoRI and BsiWI restriction sites were amplified from the SUN1-GFP plasmid (a gift of Jeremy Nathans, Johns Hopkins University, Baltimore, MD, USA) with the primers MfeI-Sun1-F and BsiW1-Sun1-R and subcloned into the vector pKT2/Fah-mCa//SB to generate pKT2/Fah-Sun1-Gfp//SB. Endotoxin-free Maxi-scale DNA extraction and purification were performed with the GenElute HP Plasmid Maxiprep Kit (MilliporeSigma).

Mouse experiments. Fah ${ }^{-1}$ mice were maintained on NTBC (Swedish Orphan Biovitrum) in the drinking water $(7.5 \mathrm{mg} / \mathrm{l})$ until hydrodynamic tail-vein injection (15) of $10 \mu \mathrm{g}$ plasmid, as specified below. For the TRAP-seq study, pKT2/Fah-Gfp-L10a//SB was injected, and the mice were euthanized 1 week $(n=3)$ or 4 weeks $(n=9)$ after injection. Likewise, for the overexpression assay, mice were injected with equimolar amounts of the plasmids pKT2/Fah-Gfp//SB and pKT2/FahSlc7a11//SB $(n=3)$ or pKT2/Fah-Slc7a11-HA//SB $(n=5)$ and euthanized 4 weeks after injection. For the CRISPR/Cas9 studies, Mice were injected with either a mixture of 10 pKT2/Fah-sgSlc7a11//SB $(n=4)$ or pKT2/Fah-sgCtl//SB $(n=4)$ in conjunction with $1 \times 10^{12}$ genome copies of AAV8.SaCas9 (Penn Vector Core) for 4 weeks of repopulation. For the ATAC-seq assay, mice were injected with pKT2/Fah-Sun1-Gfp// SB $(n=4)$. One week after plasmid injection, the livers were harvested, and GFP-positive nuclei were isolated by FACS. Mouse weights were measured 3 times per week over the course of the repopulation period to ensure successful liver regeneration. Rosa ${ }^{\text {LSL-GFP-L10a }}$ mice were purchased from The Jackson Laboratory and used as a healthy control $(n=4)$ in the TRAP-seq study, and the Ros $a^{\mathrm{LSL}-\text { Sun1-GFP }}$ mice were provided by Mitchell Lazar (University of Pennsylvania, Philadelphia, PA, USA) as a quiescent control in the ATAC-seq $(n=2)$ experiments. AAV8. TBG.PI.Cre.rBG (Penn Vector Core) was injected into the tail vein of mice at $1 \times 10^{11}$ virus particles per mouse. Mice were euthanized after 1 week of injection, and the livers were harvested. All animal studies were performed in 8- to 12-week-old female mice.

Translating RNA isolation. RNA specific for repopulating hepatocytes was isolated by TRAP (17). Briefly, $200 \mathrm{mg}$ liver tissue was taken en bloc from mice injected with the TRAP construct and from Ros $a^{\text {LSL-GFP-L10a }}$ mice, homogenized with lysis buffer, and incubated with magnetic beads that were conjugated with anti-GFP antibodies (clones Htz-GFP-19F7 and Htz-GFP-19C8, Memorial Sloan-Kettering Monoclonal Antibody Facility, New York, New York, USA) to affinity purify RNA that was bound by the GFP-L10a fusion protein.

$I H C$ and IF. Liver lobes were dissected from mice and fixed with $4 \%$ paraformaldehyde, embedded in paraffin, and sectioned. For IHC, slides were rehydrated and subjected to antigen retrieval in sodium citrate ( $\mathrm{pH}$ 6.0). $\mathrm{H}_{2} \mathrm{O}_{2}$ (30\%) was used for quenching endogenous peroxidases, and avidin $\mathrm{D}$ and biotin (Vector Laboratories) were used for blocking before incubation with primary antibodies overnight at $4^{\circ} \mathrm{C}$. The slides were then incubated with biotin-conjugated secondary antibody at $37^{\circ} \mathrm{C}$ for 30 minutes. The avidin-peroxidase complex was incubated at $37^{\circ} \mathrm{C}$ for 30 minutes (VECTASTAIN Elite Kit, Vector Laboratories). A DAB Substrate Kit for Peroxidase (Vector Laboratories) was used for development and hematoxylin for counterstaining. For IF, slides were prepared as described above. Incubation with primary antibodies was done overnight at $4^{\circ} \mathrm{C}$ in a humid chamber, followed by secondary antibody incubation for 2 hours at room temperature.
Antibodies. GFP was detected with goat anti-GFP antibody (ab6673, 1:100, Abcam) for IHC and chicken anti-GFP antibody (GFP1020, 1:300, Aves Labs) for IF staining. We used rabbit anti-mouse Ki67 antibody (SP6, 1:300, Thermo Fisher Scientific) and anti-mouse Ki67 antibody (550609, 1:200, BD Biosciences) to detect proliferating cells, rabbit anti-HA antibody (sc-805, 1:100, Santa Cruz Biotechnology) for Slc7a11-HA-positive hepatocytes, rabbit anti-mouse FAH antibody (ab81087, 1:500 for IHC and 1:200 for IF, Abcam), and DAPI for nuclear staining.

RNA-seq. RNA integrity was measured using an Agilent RNA 6000 Bioanalyzer (Agilent Technologies). cDNA libraries were made from isolated RNA with a NEBNext Ultra RNA Library Prep Kit for Illumina (New England BioLabs) according to the manufacturer's instructions. Library quality was measured with an Agilent High Sensitivity DNA Bioanalyzer, and cDNA libraries were purified and qPCR quantified (Kapa Biosystems). Twenty samples of equimolar libraries were pooled and sequenced with an Illumina HiSeq 2500.

RNA-seq data analysis. Fastq files of RNA-seq were processed using the RUM algorithm (65), with support from the University of Pennsylvania's Next Generation Sequencing Core (https://ngsc.med. upenn.edu). Differential gene expression analysis was performed using the package edgeR (66) in R software. Differentially expressed genes were identified with a cutoff of greater than 2-fold change and an FDR of less than 5\%. Congruent genes in $\mathrm{Fah}^{-/-}$and PHx models were defined as genes regulated in the same direction for at least 1 time point in both models. Quantile-normalized reads were used for generating the heatmaps with the R package aheatmap, and Venn diagrams were created using Vennerable. Gene ontology was performed using the Database for Annotation, Visualization, and Integrated Discovery (DAVID) (29,30). The top 3,000 upregulated and downregulated genes were uploaded to DAVID and analyzed using the functional annotation tool. A list of enriched KEGG pathways was obtained from the functional annotation chart report. The top-10 most significantly enriched KEGG pathways were selected and sorted according to the Bonferroni-corrected $P$ value. In addition, all differentially expressed genes, along with their corresponding fold change, were uploaded into the Ingenuity Pathway Analysis tool, and functional analysis was performed using the Core Analysis function. The upstream regulators predicted by Ingenuity Pathway Analysis were further filtered by: (a) genes that were also changed in the RNA-seq analysis by at least 2-fold; (b) a significant $Z$-score ( $\geq 2$ for predicted activation and $\leq 2$ for predicted inhibition); and (c) congruence between the observed fold change and the predicted activation or inhibition.

qRT-PCR. Extracted RNAs were reverse transcribed to cDNA with SuperScript II Reverse Transcriptase (Invitrogen, Thermo Fisher Scientific), and qRT-PCR was performed with Slc7a11 primers (Slc7a11-qRTPCR-F and -R), Gsta1 primers (Gsta1-qRTPCR-F and -R), and $T b p$ primers (Tbp-qRTPCR-F and -R). Relative expression levels were normalized to $T b p$.

qPCR. Genomic DNA was extracted from mice injected with equimolar amounts of pKT2/Fah-Gfp//SB (Fah-Gfp) and pKT2/FahSlc7a11-HA//SB (Fah-Slc7a11-HA) over a 4-week period with a DNeasy Blood and Tissue Kit (QIAGEN), followed by ethanol precipitation. qPCR was performed with PrimeTime primer sets (IDT DNA) Slc7a11 (Slc7a11-qPCR-F, -R, and -P) and Gfp (Gfp-qPCR-F, -R, and -P). Standard curves were generated by performing a serial dilution of the input plasmid with equimolar amounts of Fah-Gfp and Fah-Slc7a11-HA. 
Western blotting. Proteins were extracted from whole-liver homogenate with lysis buffer containing $50 \mathrm{mM}$ Tris, $\mathrm{pH}$ 7.5, 0.5 mM EDTA, $150 \mathrm{mM} \mathrm{NaCl}, 10 \%$ glycerol, $1 \% \mathrm{NP}-40$, and 1\% SDS, supplemented with 1:100 Halt Protease Inhibitor Cocktail (Thermo Fisher Scientific). The lysates were sonicated at 30-second intervals for 5 minutes and electrophoresed on $4 \%$ to $12 \%$ NuPAGE Precast Gels (Life Technologies, Thermo Fisher Scientific). A nitrocellulose membrane was used for transfer, and 5\% milk in TBST (TBS plus 0.1\% Tween-20) was used to block the membrane at room temperature for 1 hour. The antimouse xCT antibody (sc-79360, 1:200, Santa Cruz Biotechnology) was diluted in 5\% BSA in TBST and incubated overnight at $4^{\circ} \mathrm{C}$. The membrane was washed with TBST 3 times for 10 minutes, followed by an HRP-conjugated secondary antibody in 5\% milk in TBST for 1 hour, and then exposed to film.

Hepatocyte nuclei isolation. Livers were harvested and nuclei isolation was performed as previously described (67). Briefly, liver was dounced in a pestle tissue grinder in $10 \mathrm{ml}$ hypotonic buffer $(10 \mathrm{mM}$ Tris- $\mathrm{HCl}$, pH7.5, $2 \mathrm{mM} \mathrm{MgCl}$, $3 \mathrm{mM} \mathrm{CaCl}_{2}$ ) on ice. The homogenate was passed through a $100-\mu \mathrm{m}$ filter and sedimented at $400 \mathrm{~g}$ at $4^{\circ} \mathrm{C}$ for 10 minutes. The pellet was resuspended in $10 \mathrm{ml}$ hypotonic buffer with $10 \%$ glycerol, and $10 \mathrm{ml}$ lysis buffer (hypotonic buffer, $10 \%$ glycerol, 1\% IGEPAL CA-630) was added dropwise. After 5 minutes of incubation, the solution was centrifuged at $600 \mathrm{~g}$ for 5 minutes at $4^{\circ} \mathrm{C}$. The isolated nuclei were washed again in lysis buffer, and nuclei were counted in a hemocytometer.

Hepatocyte nuclei sorting. Isolated hepatocyte nuclei were labeled with an Alexa Fluor 647 anti-GFP antibody (338006, clone FM264G, $1: 25$, BioLegend). Immediately before cell sorting, the nuclei suspension was stained with $2 \mu \mathrm{g} / \mathrm{ml}$ DAPI. GFP-and AF647-double-positive nuclei were sorted using a BD FACSAria II, after gating for DAPI-positive nuclei. Because of the polyploidy state of the hepatocytes, only $4 n$ nuclei were collected.

ATAC-seq. Sorted hepatocyte nuclei were tagmented and PCR amplified according to a previously published ATAC-seq protocol (54, 55). Briefly, 25,000 nuclei were aliquoted, and transposition was performed at $37^{\circ} \mathrm{C}$ for 30 minutes. The transposition reaction was stopped by Buffer ERC (QIAGEN), and DNA was purified using the QIAGEN MinElute Reaction Cleanup Kit. Genomic fragments were preamplified for 5 cycles, and the final amplification cycle was determined by qPCR. The libraries were size selected with Agencourt AMPure XP beads (Beckman Coulter) and sequenced with an Illumina HiSeq 4000.

ATAC-seq data analysis. Fastq files from ATAC-seq were analyzed with the pipeline developed by Anshul Kundaje (Stanford University, Stanford, CA, USA) (https://github.com/kundajelab/atac_dnase_pipelines). Briefly, for each sample, adapters were trimmed and aligned to the genome $\mathrm{mm} 9$ with Bowtie. The aligned bam files of biological replicates were then merged and subjected to peak calling of open chromatin regions. The parameters for the analysis were -auto_detect_adapter -enable_idr -filt_bam-sample1 -filt_bam-sample2 ... -filt_bam-sampleN.

ChIP-qPCR. The Slc7a11 promoter was analyzed, and potential NRF2- and ATF4-binding motifs were identified with JASPAR (68). Liver chromatin was prepared as previously described (69). Briefly,
$100 \mathrm{mg}$ liver was fixed and sonicated with a Bioruptor (Diagenode) for 2 rounds of 7.5 minutes each. Sheared DNA $(10 \mu \mathrm{g})$ was then incubated with anti-ATF4 antibody (D4B8, 1:200, Cell Signaling Technology) and NRF2 antibodies (D1Z9C, 1:100, Cell Signaling Technology). Immunoprecipitated DNA was then isolated with phenol:chloroform extraction and subjected to qPCR analysis with the primers Slc7a11Nrf2/Atf4-ChIP-qPCR-2F and -2R. Fold enrichment was calculated by normalization to the average Ct value of Ins (Ins-ChIP-qPCR-F and -R) and Arbp (Arbp-ChIP-qPCR-F and -R) compared with input DNA.

Sequencing data download. TRAP-seq and ATAC-seq data have been deposited according to MINSEQE standards in the NCBI's Gene Expression Omnibus database (GEO GSE109466) (70). RNA-seq data from PHx are available in the ArrayExpress database (accession no. E-MTAB-1612) (71).

Statistics. Unless otherwise indicated, a 2-tailed, 2-sample Student's $t$ test was used to analyze the experimental and control groups in all assays performed in this study. A 2-tailed, 1-sample Student's $t$ test was used to compare the ratio of Fah-Slc7a11 to Fah-Gfp plasmids after liver repopulation with the injected plasmid mix. A hypergeometric test was used to analyze the overlapping genes in the scRNA-seq and TRAP-seq experiments. The Wilcoxon rank-sum test was used to compare the differential binding of NRF2 and ATF4 in the repopulating and quiescent livers. A $P$ value or FDR of less than 0.05 was considered significant. Individual data are presented as dot plots, with the mean shown as a horizontal line.

Study approval. All animal studies were reviewed and approved by the IACUC of the Penn Office of Animal Welfare (University of Pennsylvania).

\section{Author contributions}

KJW incorporated the TRAP assay into the $\mathrm{Fah}^{-/}$mouse experiments, conducted all mouse experiments, and wrote the manuscript. AWW conducted the TRAP-seq experiments and the functional studies of Slc7a11 and wrote the manuscript. YJW performed the hepatocyte nuclear isolation experiments and the ATAC-seq assay. AMZ assisted with the experimental design, edited the manuscript, and performed statistical analysis. NGM performed the IHC and IF staining in the CRISPR studies. NE maintained the $\mathrm{Fah}^{-/-}$mice. KHK directed the study and edited the manuscript.

\section{Acknowledgments}

This work was supported by the following grants: R01-DK102667 (KHK), K08-DK106478 (KJW), P30-DK050306 (KJW), T32GM008076 (to AWW), and F31-DK113666 (AWW). We thank Zhixun Dou for technical help with Western blot analysis, Shilpa Rao for RNA-seq analysis, and Yong Hoon Kim for help with the INTACT protocol and for providing the Rosa $a^{\mathrm{LSL}-\mathrm{Sun1}-\mathrm{GFP}}$-transgenic mice and ChIP-qPCR reagents.

Address correspondence to: Klaus Kaestner, Department of Genetics, University of Pennsylvania, 12-126 SCTR, 3400 Civic Center Boulevard, Philadelphia, Pennsylvania 19104 USA. Phone: 215.898.8759; Email: kaestner@mail.med.upenn.edu.
1. Lee WM. Etiologies of acute liver failure. Semin Liver Dis. 2008;28(2):142-152.

2. Taub R. Liver regeneration: from myth to mechanism. Nat Rev Mol Cell Biol. 2004;5(10):836-847.
3. Michalopoulos GK, DeFrances MC. Liver regeneration. Science. 1997;276(5309):60-66.

4. Malato Y, et al. Fate tracing of mature hepatocytes in mouse liver homeostasis and regenera- tion. J Clin Invest. 2011;121(12):4850-4860.

5. Michalopoulos GK. Liver regeneration after partial hepatectomy: critical analysis of mechanistic dilemmas. Am J Pathol. 2010;176(1):2-13. 
6. Fausto N. Liver regeneration and repair: hepatocytes, progenitor cells, and stem cells. Hepatology. 2004;39(6):1477-1487.

7. White P, Brestelli JE, Kaestner KH, Greenbaum LE. Identification of transcriptional networks during liver regeneration. J Biol Chem. 2005;280(5):3715-3722.

8. Locker J, et al. A common set of immediate-early response genes in liver regeneration and hyperplasia. Hepatology. 2003;38(2):314-325.

9. Arai M, et al. Gene expression profiling reveals the mechanism and pathophysiology of mouse liver regeneration. J Biol Chem. 2003;278(32):29813-29818.

10. Jung JW, et al. Gene expression analysis of peroxisome proliferators- and phenytoin-induced hepatotoxicity using cDNA microarray. J Vet Med Sci. 2004;66(11):1329-1333.

11. Craig A, et al. Systems toxicology: integrated genomic, proteomic and metabonomic analysis of methapyrilene induced hepatotoxicity in the rat. J Proteome Res. 2006;5(7):1586-1601.

12. Blomme EA, Yang Y, Waring JF. Use of toxicogenomics to understand mechanisms of drug-induced hepatotoxicity during drug discovery and development. Toxicol Lett. 2009;186(1):22-31.

13. Duncan AW, et al. Aneuploidy as a mechanism for stress-induced liver adaptation. J Clin Invest. 2012;122(9):3307-3315.

14. Grompe M, et al. Loss of fumarylacetoacetate hydrolase is responsible for the neonatal hepatic dysfunction phenotype of lethal albino mice. Genes Dev. 1993;7(12A):2298-2307.

15. Wangensteen KJ, et al. A facile method for somatic, lifelong manipulation of multiple genes in the mouse liver. Hepatology. 2008;47(5):1714-1724.

16. Wangensteen KJ, Zhang S, Greenbaum LE, Kaestner KH. A genetic screen reveals Foxa3 and TNFR1 as key regulators of liver repopulation. Genes Dev. 2015;29(9):904-909.

17. Heiman M, Kulicke R, Fenster RJ, Greengard P, Heintz N. Cell type-specific mRNA purification by translating ribosome affinity purification (TRAP). Nat Protoc. 2014;9(6):1282-1291.

18. Lu SC. Regulation of glutathione synthesis. $\mathrm{Mol}$ Aspects Med. 2009;30(1-2):42-59.

19. Lo M, Wang YZ, Gout PW. The x(c)-cystine/ glutamate antiporter: a potential target for therapy of cancer and other diseases. JCell Physiol. 2008;215(3):593-602.

20. Montini E, et al. In vivo correction of murine tyrosinemia type I by DNA-mediated transposition. Mol Ther. 2002;6(6):759-769.

21. Liu J, et al. Cell-specific translational profiling in acute kidney injury. J Clin Invest. 2014;124(3):1242-1254.

22. Yanger K, et al. Adult hepatocytes are generated by self-duplication rather than stem cell differentiation. Cell Stem Cell. 2014;15(3):340-349.

23. Shin S, et al. Genetic lineage tracing analysis of the cell of origin of hepatotoxin-induced liver tumors in mice. Hepatology. 2016;64(4):1163-1177.

24 . Huang P, et al. Induction of functional hepatocyte-like cells from mouse fibroblasts by defined factors. Nature. 2011;475(7356):386-389.

25. Liu Q, Yuan B, Lo KA, Patterson HC, Sun Y, Lodish HF. Adiponectin regulates expres- sion of hepatic genes critical for glucose and lipid metabolism. Proc Natl Acad Sci USA. 2012;109(36):14568-14573.

26. Severgnini M, et al. A rapid two-step method for isolation of functional primary mouse hepatocytes: cell characterization and asialoglycoprotein receptor based assay development. Cytotechnology. 2012;64(2):187-195.

27. Takahara Y, et al. Gene expression profiles of hepatic cell-type specific marker genes in progression of liver fibrosis. World J Gastroenterol. 2006;12(40):6473-6499.

28. Ju C, Tacke F. Hepatic macrophages in homeostasis and liver diseases: from pathogenesis to novel therapeutic strategies. Cell Mol Immunol. 2016;13(3):316-327.

29. Huang da W, Sherman BT, Lempicki RA. Systematic and integrative analysis of large gene lists using DAVID bioinformatics resources. Nat Protoc. 2009;4(1):44-57.

30. Huang da W, Sherman BT, Lempicki RA. Bioinformatics enrichment tools: paths toward the comprehensive functional analysis of large gene lists. Nucleic Acids Res. 2009;37(1):1-13.

31. Marí M, Colell A, Morales A, von Montfort C Garcia-Ruiz C, Fernández-Checa JC. Redox control of liver function in health and disease. Antioxid Redox Signal. 2010;12(11):1295-1331.

32. Columbano A, Shinozuka H. Liver regeneration versus direct hyperplasia. FASEB J. 1996;10(10):1118-1128.

33. Schmiedeberg P, Biempica L, Czaja MJ. Timing of protooncogene expression varies in toxin-induced liver regeneration. JCell Physiol. 1993;154(2):294-300.

34. Wangensteen KJ, et al. Combinatorial genetics in liver repopulation and carcinogenesis with a novel in vivo CRISPR activation platform [published online ahead of print November 1, 2017]. Hepatology. https://doi.org/10.1002/hep.29626.

35. Xiang D, et al. Non-viral FoxM1 gene delivery to hepatocytes enhances liver repopulation. Cell Death Dis. 2014;5:e1252.

36. Schug J, et al. Dynamic recruitment of microRNAs to their mRNA targets in the regenerating liver. BMC Genomics. 2013;14:264.

37. $\mathrm{He} \mathrm{G}$, et al. Identification of liver cancer progenitors whose malignant progression depends on autocrine IL-6 signaling. Cell. 2013;155(2):384-396.

38. Guo L, et al. Differential gene expression in mouse primary hepatocytes exposed to the peroxisome proliferator-activated receptor alpha agonists. $B M C$ Bioinformatics. 2006;7 Suppl 2:S18.

39. He F, et al. PBK/TOPK in the differential diagnosis of cholangiocarcinoma from hepatocellular carcinoma and its involvement in prognosis of human cholangiocarcinoma. Hum Pathol. 2010;41(3):415-424.

40. Yang YF, et al. PDZ binding kinase, regulated by FoxM1, enhances malignant phenotype via activation of $\beta$-Catenin signaling in hepatocellular carcinoma. Oncotarget. 2017;8(29):47195-47205

41. Halpern KB, et al. Single-cell spatial reconstruction reveals global division of labour in the mammalian liver. Nature. 2017;542(7641):352-356.

42. Huang ZZ, et al. Mechanism and significance of increased glutathione level in human hepatocellular carcinoma and liver regeneration. FASEB J.
2001;15(1):19-21.

43. Riehle KJ, Haque J, McMahan RS, Kavanagh TJ, Fausto N, Campbell JS. Sustained Glutathione Deficiency Interferes with the Liver Response to TNF- $\alpha$ and Liver Regeneration after Partial Hepatectomy in Mice. J Liver Disease Transplant. 2013;1(2):1000105.

44. Yang Y, et al. A dual AAV system enables the Cas9-mediated correction of a metabolic liver disease in newborn mice. Nat Biotechnol. 2016;34(3):334-338.

45. Ran FA, et al. In vivo genome editing using Staphylococcus aureus Cas9. Nature. 2015;520(7546):186-191.

46. Brinkman EK, Chen T, Amendola M, van Steensel B. Easy quantitative assessment of genome editing by sequence trace decomposition. Nucleic Acids Res. 2014;42(22):e168.

47. Michalopoulos GK. Liver regeneration. J Cell Physiol. 2007;213(2):286-300.

48. Sasaki H, et al. Electrophile response element-mediated induction of the cystine/glutamate exchange transporter gene expression. J Biol Chem. 2002;277(47):44765-44771.

49. Lewerenz J, Maher P. Basal levels of eIF2alpha phosphorylation determine cellular antioxidant status by regulating ATF 4 and $\mathrm{xCT}$ expression. JBiol Chem. 2009;284(2):1106-1115.

50. Lin X, Yang H, Zhang H, Zhou L, Guo Z. A novel transcription mechanism activated by ethanol: induction of Slc7a11 gene expression via inhibition of the DNA-binding activity of transcriptional repressor octamer-binding transcription factor 1 (OCT-1). J Biol Chem. 2013;288(21):14815-14823.

51. Jiang L, et al. Ferroptosis as a p53-mediated activity during tumour suppression. Nature. 2015;520(7545):57-62.

52. Deal RB, Henikoff S. A simple method for gene expression and chromatin profiling of individual cell types within a tissue. Dev Cell. 2010;18(6):1030-1040.

53. Mo A, et al. Epigenomic signatures of neuronal diversity in the mammalian brain. Neuron 2015;86(6):1369-1384.

54. Buenrostro JD, Giresi PG, Zaba LC, Chang HY, Greenleaf WJ. Transposition of native chromatin for fast and sensitive epigenomic profiling of open chromatin, DNA-binding proteins and nucleosome position. Nat Methods. 2013;10(12):1213-1218.

55. Ackermann AM, Wang Z, Schug J, Naji A, Kaestner KH. Integration of ATAC-seq and RNA-seq identifies human alpha cell and beta cell signature genes. Mol Metab. 2016;5(3):233-244.

56. Huang ZZ, Li H, Cai J, Kuhlenkamp J, Kaplowitz $\mathrm{N}, \mathrm{Lu}$ SC. Changes in glutathione homeostasis during liver regeneration in the rat. Hepatology. 1998;27(1):147-153.

57. Bunchorntavakul C, Reddy KR. Acetaminophen-related hepatotoxicity. Clin Liver Dis. 2013;17(4):587-607, viii.

58. Kinoshita H, et al. Cystine/glutamic acid transporter is a novel marker for predicting poor survival in patients with hepatocellular carcinoma. Oncol Rep. 2013;29(2):685-689.

59. Ishimoto T, et al. CD44 variant regulates redox status in cancer cells by stabilizing the $\mathrm{xCT}$ sub- 
unit of system xc(-) and thereby promotes tumor growth. Cancer Cell. 2011;19(3):387-400.

60. Timmerman LA, et al. Glutamine sensitivity analysis identifies the $\mathrm{xCT}$ antiporter as a common triple-negative breast tumor therapeutic target. Cancer Cell. 2013;24(4):450-465.

61. Wang $\mathrm{H}$, et al. Characterization of ferroptosis in murine models of hemochromatosis. Hepatology. 2017;66(2):449-465.

62. Kang ES, et al. xCT deficiency aggravates acetaminophen-induced hepatotoxicity under inhibition of the transsulfuration pathway. Free Radic Res. 2017;51(1):80-90.

63. Bae S, Park J, Kim JS. Cas-OFFinder: a fast and versatile algorithm that searches for potential off-target sites of Cas9 RNA-guided endonucle- ases. Bioinformatics. 2014;30(10):1473-1475.

64. Hough SH, Ajetunmobi A, Brody L, Humphryes-Kirilov N, Perello E. Desktop Genetics. Per Med. 2016;13(6):517-521.

65. Grant GR, et al. Comparative analysis of RNA-Seq alignment algorithms and the RNASeq unified mapper (RUM). Bioinformatics. 2011;27(18):2518-2528.

66. Robinson MD, McCarthy DJ, Smyth GK. edgeR: a Bioconductor package for differential expression analysis of digital gene expression data. Bioinformatics. 2010;26(1):139-140.

67. Kim YH, Marhon SA, Zhang Y, Steger DJ, Won KJ, Lazar MA. Rev-erb $\alpha$ dynamically modulates chromatin looping to control circadian gene transcription. Science.
2018;359(6381):1274-1277.

68. Khan A, et al. JASPAR 2018: update of the open-access database of transcription factor binding profiles and its web framework. Nucleic Acids Res. 2018;46(D1):D260-D266.

69. Bochkis IM, et al. Genome-wide location analysis reveals distinct transcriptional circuitry by paralogous regulators Foxa1 and Foxa2. PLoS Genet. 2012;8(6):e1002770.

70. Edgar R, Domrachev M, Lash AE. Gene Expression Omnibus: NCBI gene expression and hybridization array data repository. Nucleic Acids Res. 2002;30(1):207-210.

71. Kolesnikov N, et al. ArrayExpress update--simplifying data submissions. Nucleic Acids Res. 2015;43(Database issue):D1113-D1116. 